\title{
Preface: Applied Mathematical Optimization and Modelling (APMOD 2014)
}

\author{
Nalan Gülpınar ${ }^{1} \cdot$ Arne Strauss $^{1} \cdot \operatorname{Vinh}_{\text {Doan }}{ }^{1}$
}

Published online: 7 August 2017

(C) Springer Science+Business Media, LLC 2017

This issue consists of extensions of research papers presented at the "APMOD 2014, International Conference on Applied Mathematical Optimization and Modeling," 9-11 April, 2014, that was organized by the Operational Research and Management Sciences (ORMS) Group at Warwick Business School, The University of Warwick.

APMOD 2014 was the eleventh conference in the series of successful events that brought together distinguished researchers and practitioners from academia and industry to exchange knowledge, ideas, and results in a broad range of topics relevant to mathematical optimization, modelling languages and software, theory and practice of computational methods, and solution algorithms. The relevant application areas included, but were not restricted to health, transportation, logistics, engineering, finance, supply chain management, revenue management, and energy. The methodological focus of the previous conferences was on mathematical programming including stochastic programming, and more recently also heuristics and metaheuristics. In APMOD 2014, the scope was extended to include business analytics and big data.

The APMOD 2014 scientific program consisted of 111 contributed papers in 37 sessions (with 126 participants from 15 different countries). Three internationally renowned experts in mathematical programming gave plenary talks: Warren Powell (Princeton University), Berc Rustem (Imperial College London), and Stein W. Wallace (Norwegian School of Economics.

In this issue, nine selected papers reflect the diversity of applications of mathematical optimization, which is the main focus of APMOD conferences. Aouadni and Rebai introduce a decision support system to measure job satisfaction by using a genetic algorithm and multi-criteria satisfaction analysis. Bjerring, Ross, and Weissensteiner discuss feature selection for portfolio optimization. Burkart, Nolz, and Gutjahr model beneficiaries' choices in disaster relief logistics. Fokkink, Kikuta, and Ramsey study the search value of a set. Krivulin investigates the project scheduling problem using the framework of tropical optimization. Laesanklang and Landa-Silva propose new decomposition techniques with mixed integer programming and heuristics for home healthcare planning. Mohr and

Nalan Gülpınar

Nalan.Gulpinar@wbs.ac.uk

1 Warwick Business School, The University of Warwick, Coventry, UK 
Dochow introduce universal portfolios by taking into account trading risks in developing algorithms for on-line portfolio selection. Ruiz-Hernández, Elizalde, and Delgado-Gómez consider Cournot-Stackelberg games in competitive delocation. Sharma and Mehra present financial analysis based on sectoral portfolio optimization.

Finally, we would like to thank Professor E. Boros for providing us with the opportunity to act as guest-editors of the Annals of Operations Research special issue devoted to APMOD 2014. We also thank the staff members, in particular the Managing Editor, Katie D'Agosta, for her valuable support in preparing this issue. 